\title{
INFLUENCE OF PROTEIN VIBRATIONS ON ELECTRON TRANSFER
}

\author{
M. BABINCOVÁ AND P. BABINEC \\ Department of Biophysics and Chemical Pliysics, Comenius University \\ MFF UK, Mlynska Dolina, 84215 Bratislava, Slovak Republic
}

(Received April 1, 1993)

\begin{abstract}
Using a double well model complemented with the third excited state it is shown that a new excited-state-induced protein vibration-mediated channel, with an electron transfer rate comparable to a standard tunneling rate may exist. This channel can become dominant at low temperatures.
\end{abstract}

PACS numbers: $82.20 . R p, 34.70 .+\mathrm{e}, 87.15 . \mathrm{Da}$

Electron transfer in protein is one of the most important processes in living systems [1]. Many aspects of this process can be explained using e.g. molecular dynamic simulation. Since neither the quantum nature of nuclear motion nor the tunneling process can be directly described through molecular dynamics simulations, one may resort to a model, which entails the essentials of nuclear-electronic coupling and is simple enough to be amenable to a quantum mechanical analysis. The first step in this direction is a double well model [1-4, 9].

The electron transfer IIamiltonian based on the double wcll model in the Born-Oppenheimer and Condon approximations is

$$
H=-\frac{1}{2} \hbar \omega_{0} \sigma_{x}+\hbar \sum_{i} g_{i} \sigma_{z}\left(b_{i}+b_{i}^{+}\right)+\sum_{i} \hbar \omega_{i} b_{i}^{+} b_{i},
$$

where

$$
\sigma_{z}=a_{1}^{+} a_{1}-a_{2}^{+} a_{2}, \quad \sigma_{x}=a_{1}^{+} a_{2}+a_{2}^{+} a_{1} .
$$

IIerc the protein vibrations are modelled by harmonic phonons with frequencies $\omega_{i}$ and creation (annihilation) operators $b_{i}^{+}\left(b_{i}\right)$. The second term on the right hand side of (1) is the simplest non-trivial form of the electron-phonon coupling. The latter term causes the direct hopping of the electron between the states $|1\rangle=a_{1}^{+}|0\rangle$ and $|2\rangle=a_{2}^{+}|0\rangle$. If the coupling constants $g_{i}$ were zero, the Hamiltonian (1) could be exactly diagonalized, $\hbar \omega_{0}$ would be then the energy splitting between the electron eigenstates

$$
|+\rangle=\frac{1}{\sqrt{2}}(|1\rangle+|2\rangle), \quad|-\rangle=\frac{1}{\sqrt{2}}(|1\rangle-|2\rangle) .
$$


The state $|+\rangle$ may describe a neutral donor-acceptor complex DA, and the state $|-\rangle$ the ionized $\mathrm{D}^{+} \mathrm{A}^{-}$complex.

In Refs. [5-7] the usual two-state IIamiltonian has been complemented by the third state $|3\rangle=a_{3}^{+}|0\rangle$, which is also an eigenstate of the electron part of the Hamiltonian (1)

$$
H^{\prime}=H+\varepsilon a_{3}^{+} a_{3}+\hbar \sum_{i} \frac{k_{i}}{\sqrt{2}}\left(a_{1}^{+} a_{3}+a_{3}^{+} a_{1}+a_{2}^{+} a_{3}+a_{3}^{+} a_{2}\right)\left(b_{i}^{+}+b_{i}\right),
$$

where $k_{i}$ are coupling constants and $\varepsilon$ is energy of the third state.

Using Firsov-Lang unitary transformation

$$
U=\exp \left[-\sum_{i} \frac{g_{i}}{\omega_{i}}\left(b_{i}-b_{i}^{+}\right) \sigma_{z}\right],
$$

we get

$$
\begin{aligned}
\bar{H}= & U H^{\prime} U^{-1}=\bar{H}_{0}+\bar{V} \\
\bar{H}_{0}= & -\sum_{i} \hbar \frac{g_{i}^{2}}{\omega_{i}}\left(a_{1}^{+} a_{1}+a_{2}^{+} a_{2}\right)+\varepsilon a_{3}^{+} a_{3}+\sum_{i} \hbar \omega_{i} b_{i}^{+} b_{i}, \\
\bar{V}= & \hbar \sum_{i} \frac{k_{i}}{\sqrt{2}}\left\{a_{1}^{+} \exp \left[-\sum_{j} \frac{g_{j}}{\omega_{j}}\left(b_{j}-b_{j}^{+}\right)\right] a_{3}\left(b_{i}+b_{i}^{+}\right)\right. \\
& \left.+a_{2}^{+} \exp \left[+\sum_{j} \frac{g_{j}}{\omega_{j}}\left(b_{j}-b_{j}^{+}\right)\right] a_{3}\left(b_{i}+b_{i}^{+}\right)\right\}+ \text {herm.conj. }
\end{aligned}
$$

This Hamiltonian was studied in Refs. [5-7] via the gencralized master equation using a projector, which suppresses all the physical information connected with the third state and it was shown that when the standard tunnelling and overbarrier channels become sufficiently suppressed, the electron transfer rate becomes

$$
K=\frac{1}{\hbar \varepsilon} \sum_{\lambda, \mu, \nu} \bar{V}_{1 \nu, 3 \mu} \bar{V}_{3 \mu, 2 \lambda} \rho_{\lambda \nu}^{R}
$$

where Greek indices designate the bath states and $\rho^{R}$ is the initial bath density matrix.

Using the formula (9) we can obtain approximately the transfer rate. Here $\varepsilon$ is in the interval of $10^{-1}-10^{2} \mathrm{eV}$ [1]. Assuming the strength of the coupling to the bath as $10^{-3}-10^{-2} \mathrm{eV}$ [1] and approximating the matrix element of $\bar{V}$ as this strength multiplied by a corresponding overlap, we have

$$
K \approx 1.52 \times 10^{7} S_{1,3} S_{2,3} \text { to } 1.52 \times 10^{12} S_{1,3} S_{2,3}
$$

where overlaps $S_{1,3}, S_{2,3}$ have values $\approx 10^{-3}$ [1]. Therefore, the electron transfer rate is in the interval

$$
K \approx 1.52 \times 10^{1}-1.52 \times 10^{6} \mathrm{~s}^{-1} \text {. }
$$

This value is comparable with those obtained for the standard tunneling transfer in protein $[1,9]$. 
The aim of this letter has been to illustrate a simple model that consistent treatment of electron transfer in protein needs quantum-mechanical description of biopolymer and that approximations must be done very carefully. This excited-state-induced protein vibration-mediated cliannel can be important, e.g. in explaining an observed increase in the electron transfer rate at lower temperatures $[8,9]$.

\section{References}

[1] E.G. Petrov, Physics of Electron Transfer in Biosystems, Naukova Dumka, Kiev 1984 (in Russian).

[2] A. Garg, J.N. Onuchic, V. Ambegaokar, J. Chem. Phys. 83, 4491 (1985).

[3] C. Zheng, J.A. McCammon, P. Wolynes, Chem. Phys. 158, 261 (1991).

[4] K. Schulten, M. Tesch, Chem. Phys. 158, 421 (1991).

[5] V. Ćápek, J. Phys. (France) 50, 887 (1989).

[6] V. Cápek, Czech. J. Phys. B 39, 793 (1989).

[7] V. Ćápek, J. Phys. C 20, 2901 (1987).

[8] R.H. Austin, M.K. Hong, C. Moser, J. Plombon, Chcm. Phys. 158, 473 (1991).

[9] D. DeVault, Quantum-mechanical Tunneling in Biological Systems, Cambridge University Press, New York 1984. 